\title{
Melt-particle mixing in gas-stirred ladles with throughflow
}

\author{
S. Torii* and W.-J. Yang \\ Dept. of Mechanical Engineering and Applied Mechanics, University of Michigan, Ann Arbor, Michigan 48109, USA
}

\begin{abstract}
An experimental study is performed on a gas-particle stirred ladle system with throughflow, using a simplified water model. Narrow ladles are used to produce 2-D flows. Flow visualization by the direct photographic method is employed to investigate the effects of ladle geometry, throughflow rate, air flow rate and its injection location on the melt-particle mixing performance. Image processing is applied to aid in determining the mixing performance. It is disclosed that an efficient mixing may be achieved if the gas at a higher flow rate is injected with particles through a nozzle near the bottom corner of the ladle wall on the melt inlet side. The mixing performance is better in a rectangular ladle (aspect ratio of 2) than in a square ladle (aspect ratio of unity). The effect of throughflow rate on mixing is minor. The study has an important application in manufacturing processes, such as continuous casting process, and materials processing.
\end{abstract}

\section{List of symbols}

$A R$ aspect ratio

$B$ width of water vessel, $\mathrm{m}$

$B n$ Nozzle location on bottom surface of water vessel, $m$

$H$ height of water vessel or height between bottom surface and free surface of water vessel, $m$

$H n$ Nozzle location on vertical (inlet side) surface of water vessel, $m$

$Q_{g} \quad$ volumetric rate of gas, $\mathrm{m}^{3} / \mathrm{s}$

$Q_{l} \quad$ volumetric rate of water, $\mathrm{m}^{3} / \mathrm{s}$

$Q_{\text {s }} \quad$ volumetric rate of particle, $\mathrm{m}^{3} / \mathrm{s}$

$x$ transverse coordinate, $\mathrm{m}$

$y \quad$ longitudinal coordinate, $\mathrm{m}$

\section{Introduction}

The ladle is a device of deep-bowled long-handled geometry which is used for conveying longs such as molten metals in materials processing. Since the 1970's (Sahai and Guthrie 1986), ladle metallurgy has been firmly established as a technique for treating molten steel prior to continuous casting. Essentially, ladle metallurgical operations entail the agitation of steel held in a ladle while homogenization, bath

* Visiting scholar on leave from the Mechanical Engineering Department, Kagoshima University, Japan addition, temperature adjustment or some other operation (e.g., desulphurization, deoxidization, etc.) is taking place (for example, Szekely et al. 1976, Grevet et al. 1982, Sahai and Guthrie 1982a and b, Mazumdar and Guthrie 1985). Three fundamental techniques are available for agitation: gas bubble injection, circulation involving vacuum, and induction stirring.

In gas-stirred systems, gas is injected through a submerged lance, plug, or nozzle to stir the contents. In the course of its ascension through the liquid or melt, the gas performs mixing, promotes chemical reactions, and minimizes temperature and composition inhomogeneities. Moreover, through the generation of turbulence, it may also aid inclusion agglomeration and float-out. Typical examples of gas mixing applications include the desulphurization of iron or steel, the modification of inclusion morphology and composition by the injection of powders or pellets, and the decarburization of steel by argon in combination blowing practices.

The injection of powders into melts, which is referred to as injection metallurgy, has become of increasing importance to metallurgical refining operations. In industrial injection processes, such as the external desulphurization of molten iron by powdered calcium carbide, powders are in dense phase and are pneumatically transported, then injected into the liquid metal through a submerged lance. The carrier gas, while rising as a plume to the surface, induces circulatory flows in the fluid within the vessel, thereby helping to promote metal homogeneity.

The laboratory modeling of metal-gas flows have most frequently used water as the modeling medium. This is because the kinematic viscosity of water as the most important property is essentially equivalent to that of molten steel at 1,873 K (Sahai and Guthrie 1986). There have been numerous theoretical and experimental studies on recirculating flow in gas-stirred ladle systems and their flow characteristics. Such studies were performed by Szekely et al. (1976), Grevet et al. (1982), Sahai and Guthrie (1982a and b) and Mazumdar and Guthrie (1985). These ladle systems have no throughflow with solely gas injection. The hydrodynamics of 


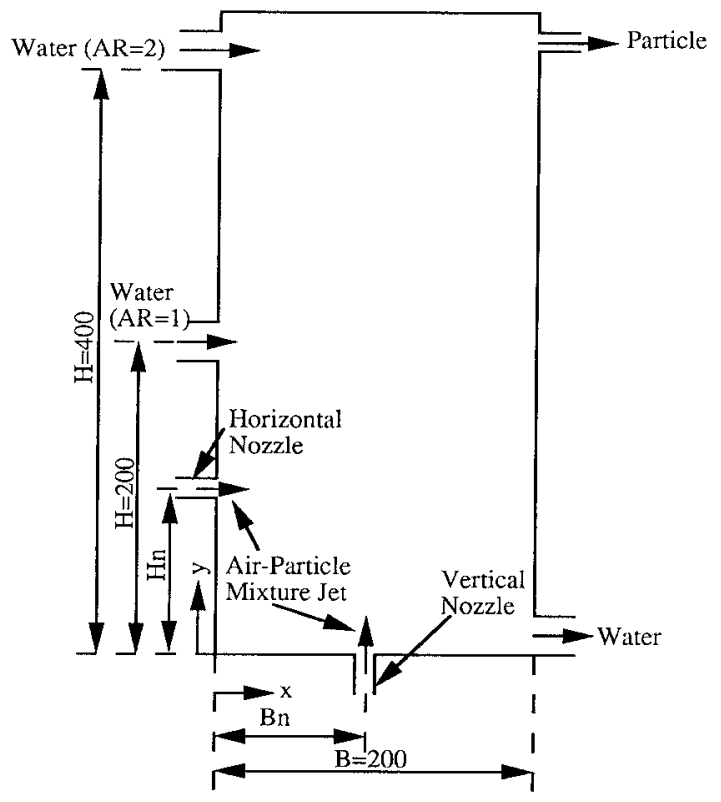

Fig. 1. A schematic of the experimental apparatus

injection metallurgy plays an important role in the kinetic efficiency of chemical reactions such as desulphurization. However, no mathematical modeling of these industrial operations has been reported, due to the difficulty of modeling the 3-D character of the flow fields. The first step to investigating such solid-gas-liquid flows is to observe simulated flows within the ladle.

The proposed study is intended to derive design guidelines for gas-powder stirred ladle systems with throughflow and to determine their mixing performances. The flow, in practice, is three-dimensional. Due to the complexity of the flow, it is very difficult to determine the operating conditions for optimum mixing performance. As a first step, a ladle is constructed to investigate the 2-D flow characteristics. Based on the results, gas-stirred solid-liquid mixing problems in 3-D systems will be formulated. The purpose of the present study is to determine the influence of ladle geometry, nozzle location and shape, melt flow rates, and flow rates of gas and particle on the flow pattern and mixing characteristics. The gas-powder mixing performance can be evaluated from photographs of the ladle flow. Image processing is applied to aid in determining the liquid-particle mixing performance.

\section{Experimental apparatus}

The experimental apparatus used in the present study is depicted in Fig. 1. It consisted of a slender rectangular tank (simulating a ladle with a 2-D flow field in the $x$ and $y$ directions) with two large side walls which were made of acrylic windows for illumination and observation. Water was used to simulate the molten metal which flowed steadily
Table $1 \mathbf{a}$ and $\mathbf{b}$. Summary of data presentation; a rectangular ladle $(A R=2)$, b Square ladle $(A R=1)$

\begin{tabular}{|c|c|c|c|c|}
\hline $\begin{array}{l}\text { Air nozzle } \\
\text { location }\end{array}$ & $\begin{array}{l}\text { Water }\left(\mathrm{m}^{3} / \mathrm{s}\right) \\
Q_{l} \times 10^{5}\end{array}$ & $\begin{array}{l}\text { Air }\left(\mathrm{m}^{3} / \mathrm{s}\right) \\
Q_{g} \times 10^{4}\end{array}$ & $\begin{array}{l}\text { Solid }\left(\mathrm{m}^{3} / \mathrm{s}\right) \\
Q_{s} \times 10^{6}\end{array}$ & $\begin{array}{l}\text { Case } \\
\text { number }\end{array}$ \\
\hline \multicolumn{5}{|l|}{$\mathbf{a}$} \\
\hline$B n / B=0.50$ & $\begin{array}{l}1.88 \\
1.84 \\
3.33 \\
3.32\end{array}$ & $\begin{array}{l}1.10 \\
2.43 \\
1.09 \\
2.44\end{array}$ & $\begin{array}{l}3.32 \\
3.32 \\
3.32 \\
3.32\end{array}$ & $\begin{array}{l}\text { A1 } \\
\text { A2 } \\
\text { A3 } \\
\text { A4 }\end{array}$ \\
\hline$B n / B=0.05$ & $\begin{array}{l}1.88 \\
1.86 \\
3.33 \\
3.34\end{array}$ & $\begin{array}{l}1.07 \\
2.41 \\
1.08 \\
2.39\end{array}$ & $\begin{array}{l}3.32 \\
3.32 \\
3.32 \\
3.32\end{array}$ & $\begin{array}{l}\text { A5 } \\
\text { A6 } \\
\text { A7 } \\
\text { A8 }\end{array}$ \\
\hline$H n / H=0.05$ & $\begin{array}{l}1.88 \\
1.89 \\
3.30 \\
3.31\end{array}$ & $\begin{array}{l}1.10 \\
2.40 \\
1.08 \\
2.42\end{array}$ & $\begin{array}{l}3.32 \\
3.32 \\
3.32 \\
3.32\end{array}$ & $\begin{array}{l}\text { A9 } \\
\text { A10 } \\
\text { A11 } \\
\text { A12 }\end{array}$ \\
\hline$H n / H=0.50$ & $\begin{array}{l}1.89 \\
1.87 \\
3.33 \\
3.35\end{array}$ & $\begin{array}{l}1.08 \\
2.44 \\
1.09 \\
2.46\end{array}$ & $\begin{array}{l}3.32 \\
3.32 \\
3.32 \\
3.32\end{array}$ & $\begin{array}{l}\text { A13 } \\
\text { A14 } \\
\text { A15 } \\
\text { A16 }\end{array}$ \\
\hline \multicolumn{5}{|l|}{ b } \\
\hline$B n / B=0.50$ & $\begin{array}{l}1.87 \\
1.88 \\
3.35 \\
3.37\end{array}$ & $\begin{array}{l}1.09 \\
2.46 \\
1.05 \\
2.43\end{array}$ & $\begin{array}{l}3.32 \\
3.32 \\
3.32 \\
3.32\end{array}$ & $\begin{array}{l}\text { B1 } \\
\text { B2 } \\
\text { B3 } \\
\text { B4 }\end{array}$ \\
\hline$B n / B=0.05$ & $\begin{array}{l}1.88 \\
1.86 \\
3.34 \\
3.36\end{array}$ & $\begin{array}{l}1.08 \\
2.47 \\
1.05 \\
2.43\end{array}$ & $\begin{array}{l}3.32 \\
3.32 \\
3.32 \\
3.32\end{array}$ & $\begin{array}{l}\text { B5 } \\
\text { B6 } \\
\text { B7 } \\
\text { B8 }\end{array}$ \\
\hline$H n / H=0.05$ & $\begin{array}{l}1.89 \\
1.88 \\
3.37 \\
3.33\end{array}$ & $\begin{array}{l}1.10 \\
2.47 \\
1.09 \\
2.41\end{array}$ & $\begin{array}{l}3.32 \\
3.32 \\
3.32 \\
3.32\end{array}$ & $\begin{array}{l}\text { B9 } \\
\text { B10 } \\
\text { B11 } \\
\text { B12 }\end{array}$ \\
\hline$H n / H=0.50$ & $\begin{array}{l}1.87 \\
1.86 \\
3.34 \\
3.33\end{array}$ & $\begin{array}{l}1.10 \\
2.44 \\
1.08 \\
2.46\end{array}$ & $\begin{array}{l}3.32 \\
3.32 \\
3.32 \\
3.32\end{array}$ & $\begin{array}{l}\text { B13 } \\
\text { B14 } \\
\text { B15 } \\
\text { B16 }\end{array}$ \\
\hline
\end{tabular}

at a volumetric rate of $Q_{l}$ into and out of the tank via the inlet and exit pipes of $10 \mathrm{~mm}$ inner diameter. A stream of air (simulating the carrier gas) $Q_{g}$, mixed with neutrally buoyant solid particles in water (simulating desulphurization or deoxidizing agent) $Q_{s}$, was injected into the tank via a submerged nozzle with a $5 \mathrm{~mm}$ inner diameter to stir the throughflow of water. The average bubble diameter exiting from the air nozzle was $25 \sim 30 \mathrm{~mm}$. The solid particles employed were polypropylene spheres $500 \mu \mathrm{m}$ in diameter. They were dispersed in the ladle, eventually arrived at the free surface in the vicinity of the verticala (exit side) wall, and flowed out of the tank into a drain pipe. The water and gas flow rates were varied, while particles were injected at a constant rate.

The location of the submerged air nozzle was varied to evaluate its effect on the liquid-particle mixing and flow turbulence in the tank. The nozzle was installed on the mid- 
point of the slender sides at various location on both the bottom and vertical (inlet side) surfaces to determine its optimum location for each combination of $Q_{g}$ and $Q_{l}$ in a given tank. Two tanks with the dimensions of $B=200 \mathrm{~mm}$ and $H=(200,400) \mathrm{mm}$ (see Fig. 1) were tested, equivalent to two different aspect ratios $(H / B=2$ and 1$)$, to determine the effect of tank geometry on the mixing performance. In the case of aspect ratio $H / B=1$, the inlet water pipe was installed at $H=200 \mathrm{~mm}$ on the vertical (inlet side) surface. The combinations of the nozzle location and the water and air flow rates are summarized in Table $1 \mathrm{a}$ and $\mathrm{b}$ for $H / B$ of 2 and 1 , respectively.

\section{Experimental procedure}

Direct photography was employed in flow visualization. The flow velocity distribution in the gas-particle mixing tank was determined by taking a single-exposed photograph over a desired time interval. The movement of each particle (tracer particle) over the exposure time was used to calculate the local velocity. For example, Fig. $2 \mathrm{a}$ is a photograph with $1 / 30 \mathrm{~s}$ exposure time of case A12. The throughflow water at $Q_{l}=3.31 \times 10^{-5} \mathrm{~m}^{3} / \mathrm{s}$ entered the tank from the upper left corner and exited at the lower right corner, while the air-particle mixture at $Q_{g}=2.42 \times 10^{-4} \mathrm{~m}^{3} / \mathrm{s}$ and $Q_{s}=3.32 \times 10^{-6} \mathrm{~m}^{3} / \mathrm{s}$ was injected into the tank via a horizontal nozzle installed at the lower left corner. White lumps show agglomeration, while white traces correspond to particle loci. By measuring the length of a trace with a known exposure time, a local flow velocity can be calculated. Care was taken to extract particle streaks such that no particles may cause agglomeration. Figure $2 \mathrm{~b}$ is a schematic of the flow pattern in Fig. 2a. This method of particulate velocimetry is favored if the particles are neutrally buoyant, such as small polypropylene spheres in water in the present case. In the present study, mixing implies a homogeneous solidliquid mixing with higher particle velocity distribution in a ladle.

The photograph was analyzed by means of digital image processing. This system consisted of a video camera with $640 \times 480$ pixels, a scanner (converting the optical image into a digitized image), a monitor, a 32-bit personal computer, and a laser printer (printing dots at $300 \mathrm{DPI}$ ). Software operations being employed included edge enhancement, reversing, posterizing, and thresholding with individual operation functioning as follows: (1) Edge enhancement: the original image was enhanced by a filter, which increased the contrast along the edges of the image; (2) Reversing: the grey shades of the original images were reversed; (3) Posterizing: the grey level of the original images was reduced to eight shades; and (4) Thresholding: the grey level image data were thresholded into binary image data, with only two gray levels of either 0 (white) or 1 (black). In the present study, edge enhancement was used predominantly in the image processing.

\section{Results and discussion}

\subsection{Case $A(H / B=2)$}

The flow patterns of cases A1, A2, A3 and A4, with the air nozzle installed at $B n / B=0.50$, exhibit vortices on both sides of the bubble column not shown. A stream of high velocity

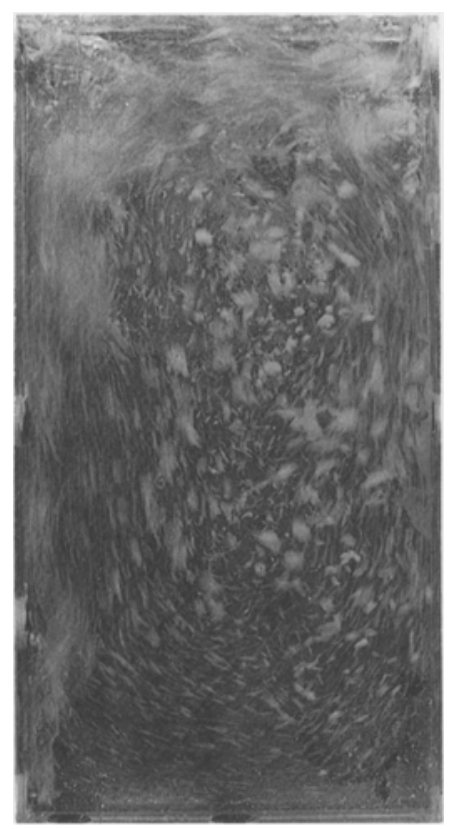

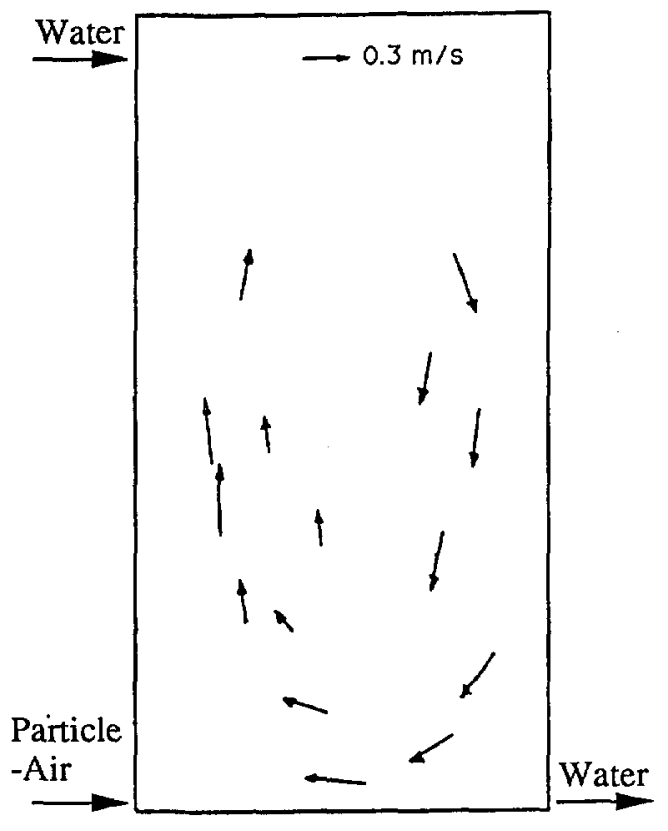

Fig. $2 a$ and b. Photograph and schematic of mixing flow in a rectangular ladle $(A R=2)$ with the air injection nozzle at $H n / H=0.05$ for case $\mathrm{A} 12$ 
liquid induced by the bubble column ascended toward the free surface, flowed downward along the vertical sidewalls, and merged into the rising bubble column. This high velocity liquid varied with a change in the air flow rate from $Q_{l}=1.09 \times 10^{-4} \mathrm{~m}^{3} / \mathrm{s}$ to $Q_{l}=2.46 \times 10^{-4} \mathrm{~m}^{3} / \mathrm{s}$, keeping the water flow rate constant. In case $\mathbf{A} 1$, one vortex formed near the water inlet and another near the water outlet, while in case A2, one vortex formed on each side of the bubble column. A similar phenomenon was observed in the higher water flow cases, A3 and A4. However, at a constant air flow rate, a change in water flow rate produced no effect on the mixing performance of the ladle. In both cases A1 and A3, lower air flow rates resulted in a curved bubble column, asymmetrical recirculation patterns, and a less efficient mixing performance. In contrast, the higher air flow rate cases, A2 and A4, yielded a straight bubble column, and consequently, nearly symmetrical recirculation patterns, and more efficient mixing characteristics.

If the air nozzle is located at $B n / B=0.05, H n / H=0.05$, or $H n / H=0.5$, only a single vortex appears in the tank. Figures $3 \mathrm{a}-\mathrm{d}$ present the schematics of the flow patterns of cases A5, A6, A7 and A8, respectively, with the air nozzle at $B n / B=0.05$. In all these cases, particles and bubbles ascended along the left sidewall and descended along the right sidewall, forming a large clockwise vortex in the tank. However, local velocities differed depending on the air flow rate. Maintaining the water flow rate constant, an increase in the air flow rate, from case $\mathrm{A} 5$ to $\mathrm{A} 6$ or from case $\mathrm{A} 7$ to $\mathrm{A} 8$, resulted in substantially enhanced local velocities, as seen in Fig. 3. Similar characteristics were observed with the air nozzle at $H n / H=0.05$. Better solid-liquid mixing was achieved in the whole region of the ladle through greater recirculation of the ascending bubbles.

For the air nozzle at $H n / H=0.5$, there appears a single vortex similar to that in Fig. 3, but of less strength. This is due to a reduction in the lance depth from $0.95 \mathrm{H}$ to $0.5 \mathrm{H}$, causing the liquid recirculation speed to decrease. Therefore, it is important to note that the vortex strength is affected by the hydrostatic pressure at the position of the air nozzle depth for a given air flow rate. In summary, better mixing is achieved by a higher flow rate or with the air nozzle located at $B n / B=0.05$ and $H n / H=0.05$.

\subsection{Case $B(H / B=1)$}

Figures for case $\mathrm{B}$ are omitted in the interest of brevity. The flow patterns in cases B1, B2, B3, and B4 for the air nozzle at $B n / B=0.5$ are similar to cases $\mathrm{A} 1, \mathrm{~A} 2, \mathrm{~A} 3$ and $\mathrm{A} 4$, with an aspect ratio of 2 . A pair of vortices appeared in the tank with an aspect ratio of unity at a high air flow rate, cases $\mathbf{B} 2$ and B4. The vortices were nearly symmetrical with respect to the bubble column. In contrast, the vortices were asymmetrical at a lower air flow rate, in cases B1 and B3. Efficient solidliquid mixing was achieved in the whole region at a relatively high air flow rate. However, both clockwise and counterclockwise circulations caused particles to congregate at the free surface near the side walls. Since the particles in the ladle must exit through the discharged pipe opposite the inlet water pipe, the air nozzle at $B n / B=0.5$ is not satisfactory for particle-liquid mixing.

The velocity profiles for the air nozzle at $B n / B=0.05$ are very smilar to those in Fig. 3. Each velocity profile consists
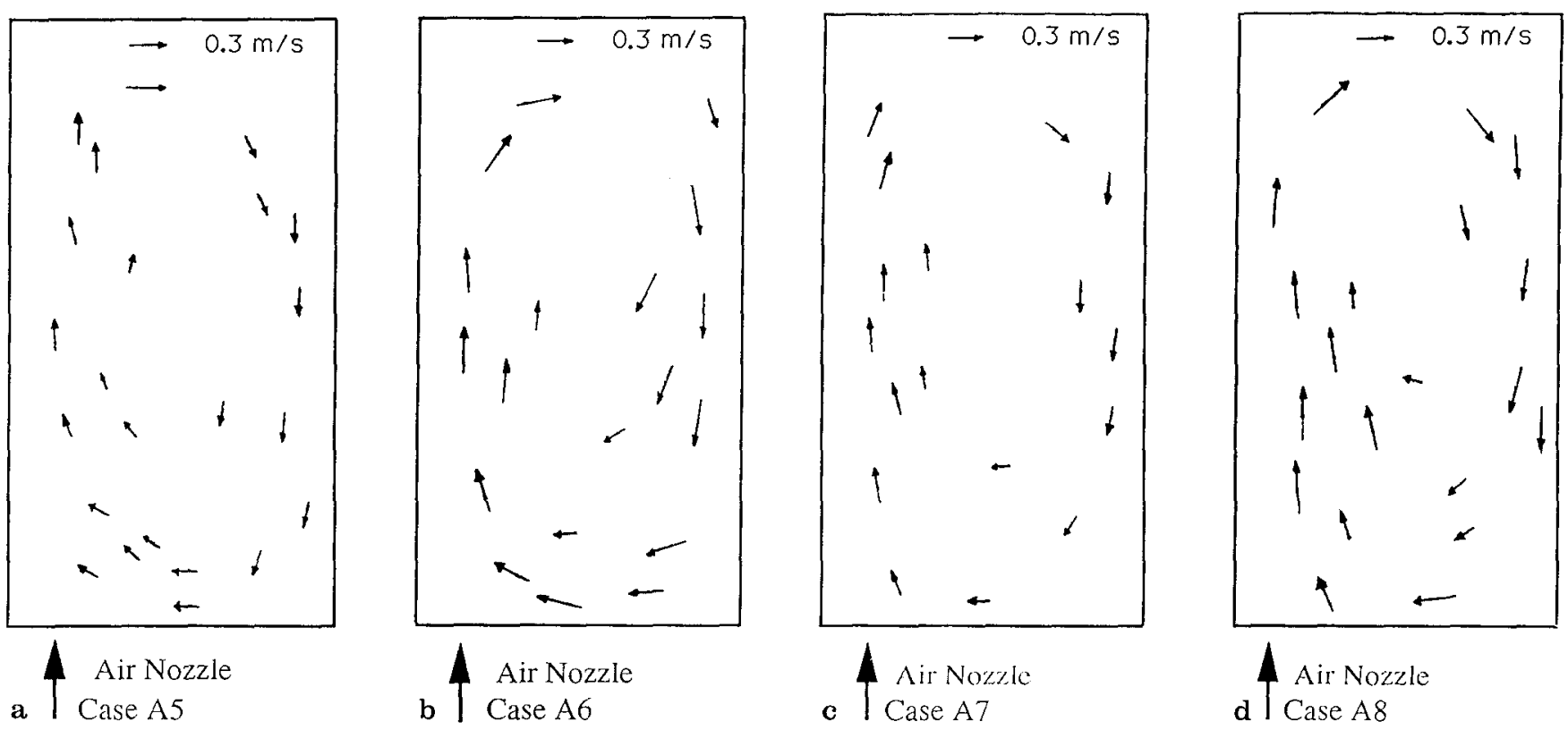

Fig. 3a-d. Schematics of mixing flow in a rectangular ladle $(A R=2)$ with the air injection nozzle at $B n / B=0.05$ for cases $A 5$ through $A 8$ 

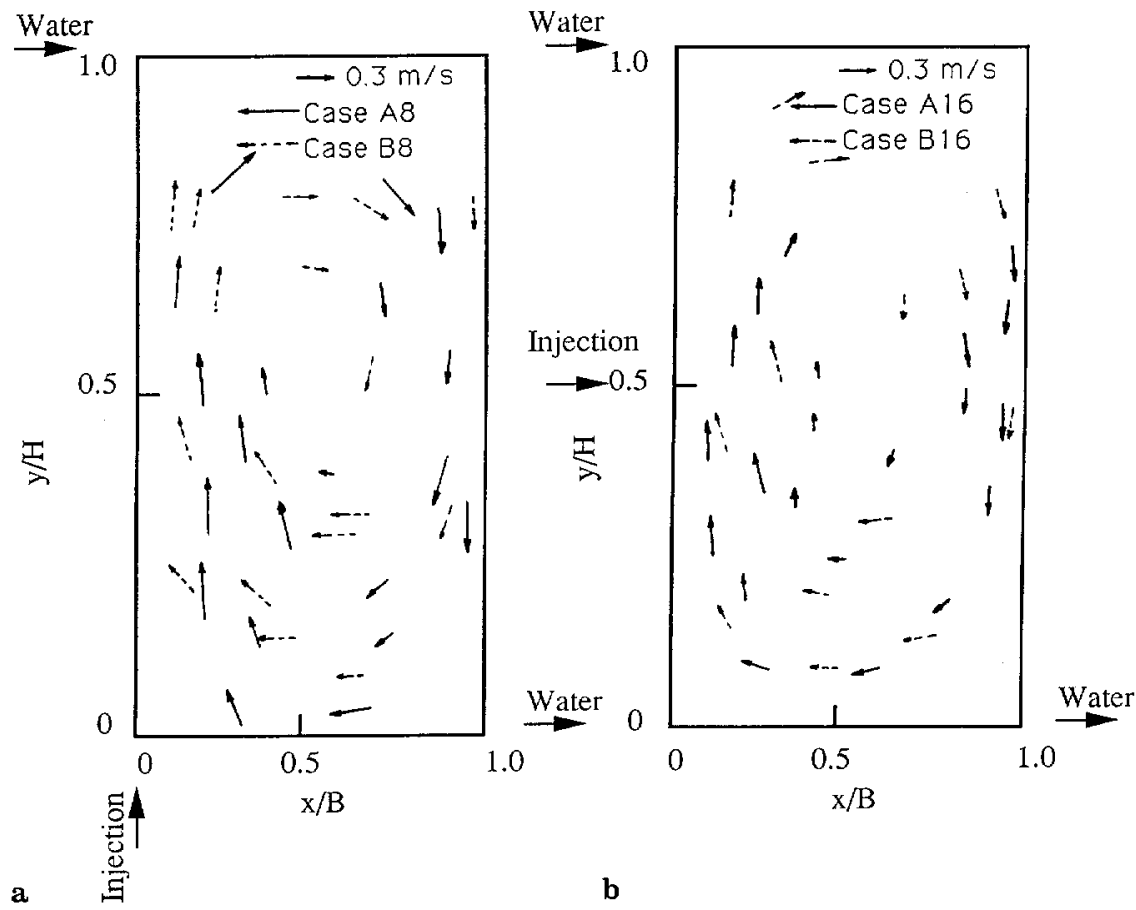

Fig. $4 a$ and b. Comparison of the velocity distributions of rectangular and square ladles for a cases $\mathrm{A} 8$ and $\mathrm{B} 8$ and $\mathrm{b}$ cases A16 and B16

of a single large clockwise recirculating flow. Cases B6 and B8, for higher air flow rates, exhibit strong vortex characteristics with rising bubbles disturbing the free surface. This recirculation induced by the higher air flow rate results in efficient solid-liquid mixing. Similar flow patterns were observed with the air nozzle at $H n / H=0.05$ and $H n / H=0.5$, respectively. With the same throughflow and air injection rates in both cases, almost the same recirculation velocities were observed with the air nozzles at $B n / B=0.05$ and $H n / H=0.05$, but with a slightly slower recirculation flow due to a reduction in the lance depth. Therefore, it is important to note that the recirculation strength depends on the depth of the air nozzle. In addition, a strong recirculation caused by the rising bubbles gives rise to better solid-liquid mixing in the entire flow field. It is concluded that a higher air flow rate from a nozzle at $B n / B=0.05$ or $H n / H=0.05$ yields a better mixing performance.

\subsection{Optimum operation in gas-powder stirred ladle systems}

The preceeding sections have presented the effects of air and water flow rates and air nozzle location on the mixing performance of ladles with two different aspect ratios. The present section investigates the influence of the ladle geometry on the mixing performance to determine the optimum operating conditions for gas-powder mixing.

Figure $4 \mathrm{a}$ replots the velocity profiles of cases $\mathrm{A} 8$ and $\mathrm{B} 8$ using solid and dotted lines, respectively. The locations of the air-particle jet and the water inlet and outlet pipes are indicated. It is demonstrated in Fig. 4 a that the flow speed

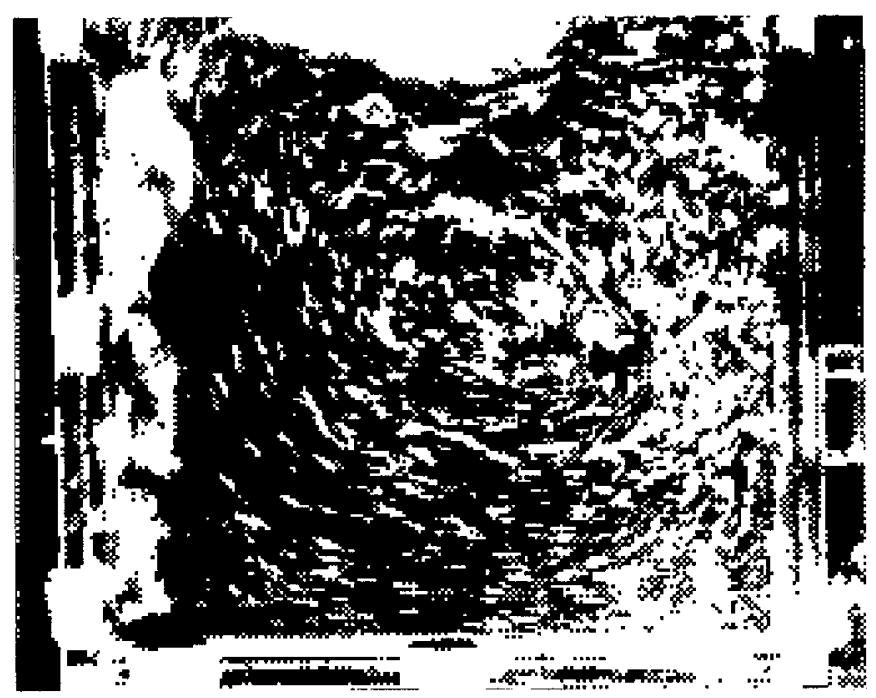

Fig. 5. Image processing by edge enhancement on the particle distribution in mixing flow inside a square ladle with the air injection nozzle at $H n / H=0.05$ for case $\mathrm{B} 11$

in case $\mathrm{A} 8$ is much higher than that in case $\mathbf{B} 8$. This is also observed in Fig. 4 b for the air nozzle at $H n / H=0.5$, but with a slight difference in flow velocities. Hence, under the same operation conditions, namely same air and water flow rates and same location of the air nozzle, case $\mathrm{A}(A R=2)$ yields a stronger recirculation flow than case $\mathrm{B}(A R=1)$.

In conclusion, a ladle with aspect ratio of $H / B=2$ and a higher air flow rate from a nozzle at $B n / B=0.05$ or $H n / H=$ 0.05 produces the best mixing performance. 


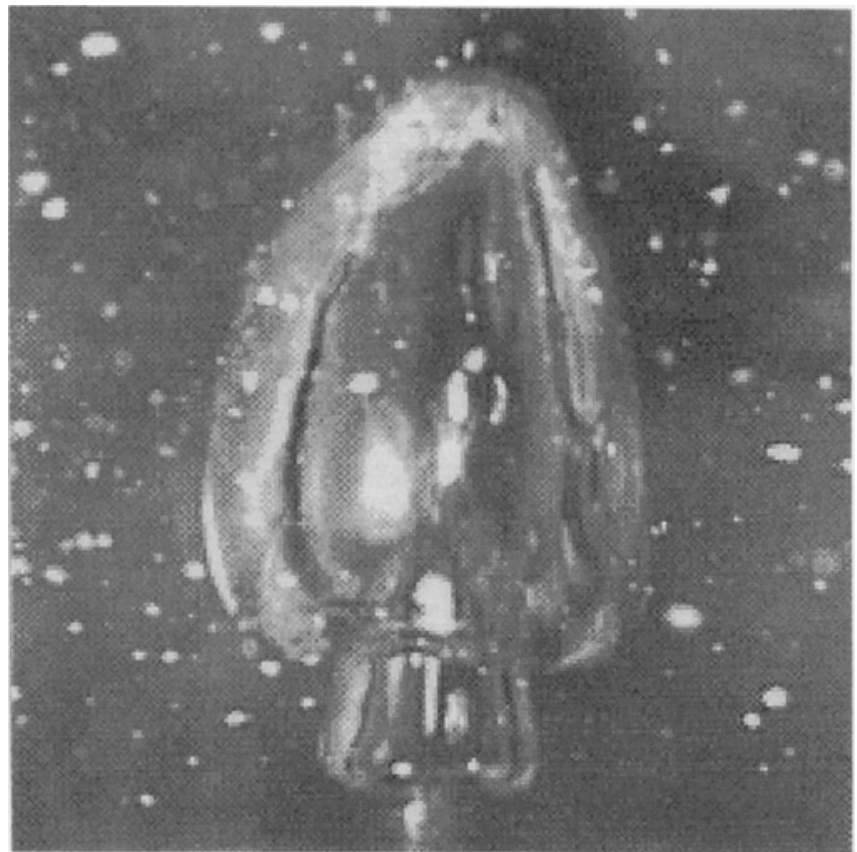

Fig. 6. Image processing by edge enhancement of the particle distribution in an air bubble exiting from an injection nozzle at $B n / B=0.5$ for a rectangular ladle for case A4

\section{Image processing}

Image processing was performed on photographs to aid in determining the liquid-particle mixing performance. Figure 5 depicts the result of image processing by edge enhancement on the particle distribution in mixing flow inside the square ladle with an air injection nozzle at $H n / H=0.05$ for case B11. Particles and their clusters appear in white color, exhibiting a homogeneous mixing. Edge enhancement is a process to accentuate edges of all particle images thus sharpening the image of particle distribution. Figure 6 shows the particle distribution in an air bubble exiting from a nozzle at $B n / B=0.05$ for case A4. A large white area on the bubble surface is due to light reflection. It is seen that some particles are attached on the bubble surface, while some are entrained inside the bubble. Results from the image processing indicate that the method is a useful aid in the investigation of liquidparticle mixing performance.

\section{Summary}

An experimental study has been conducted on the mixing characteristics of gas-powder stirred ladle systems with throughflow to determine the optimum conditions for solidliquid mixing. Two different aspect ratios are treated. The results are summarized as follows:

Efficient liquid-particle mixing is achieved at a higher air injection rate from a nozzle near the bottom corner on the vertical wall $(H n / H=0.05$ or $B n / B=0.05)$. The mixing performance is found to be more efficient in a rectangular vessel with an aspect ratio of 2 than in a square vessel with an aspect ratio of unity. The finding is supported by the results of image processing on photographs.

\section{Acknowledgements}

The presented work was supported by the National Science Foundation under the Grant Number CTS-8921584.

\section{References}

Grevet, J. H.; Szekely, J.; El-Kaddah, N. 1982: An experimental and theoretical study of gas-bubble driven circulation systems. Int. J. Heat Mass Transf. 25, 487-497

Mazumdar, D.; Guthrie, R. I. L. 1985: Hydrodynamic modeling of some gas injection procedures in ladle metallurgy operations. Metall. Trans. B. 16B, 83-90

Sahai, Y.; Guthrie, R. I. L. 1986: Recent advances in the hydrodynamics of metallurgical processing. In: Advances in transport processes. (eds. Mazumdar, A. S.; Mashelakar, R. A.). pp. 1-48. New York: Halsted Press

Sahai, Y.; Guthrie, R. I. L. 1982a: Hydrodynamics of gas stirred melts: part I. gas/liquid coupling. Metall. Trans. B. 13B, 193-202

Sahai, Y.; Guthrie, R. I. L. 1982 b: Hydrodynamics of gas stirred melts: part II. axisymmetric flows. Metall. Trans. B. 13 B, 203211

Szekely, J; Wang, H. J.; Kiser, K. M. 1976: Flow pattern velocity model of an argon-stirred ladle. Metall. Trans. B. 7 B, 287-295

Received September 16, 1991 